$\xi=\mathbf{Z}$

\title{
On HPM approximation for the perihelion precession angle in general relativity
}

\author{
V. K. Shchigolev *, D. N. Bezbatko \\ Department of Theoretical Physics, Ulyanovsk State University, Ulyanovsk, 432000, Russia \\ *Corresponding author E-mail: vkshch@yahoo.com
}

\begin{abstract}
In this paper, the homotopy perturbation method (HPM) is applied for calculating the perihelion precession angle of planetary orbits in General Relativity. The HPM is quite efficient and is practically well suited for use in many astrophysical and cosmological problems. For our purpose, we applied HPM to the approximate solutions for the orbits in order to calculate the perihelion shift. On the basis of the main idea of HPM, we construct the appropriate homotopy that leads to the problem of solving the set of linear algebraic equations. As a result, we obtain a simple formula for the angle of precession avoiding any restrictions on the smallness of physical parameters. First of all, we consider the simple examples of the Schwarzschild metric and the Reissner - Nordström spacetime of a charged star for which the approximate geodesics solutions are known. Furthermore, the implementation of HPM has allowed us to readily obtain the precession angle for the orbits in the gravitational field of Kiselev black hole.
\end{abstract}

Keywords: General Relativit; Homotopy Perturbation Method; Perihelion Precession; Kiselev Black Hole.

\section{Introduction}

The shift of the perihelion of planetary orbits is a classical test of any theory of gravity [1], [2]. For example, the perihelion precession in General Relativity (GR) and some modified theories of gravity, were recently considered in [3]-[8]. The perturbations determined by a generic alternative theory of gravity to the GR solution describing the gravitational field around a central mass are worked out in [9]. The authors of [10] used recent observations from solar system orbital motions in order to constrain some alternative theories of gravitation. In particular, the spherical solutions of the theory are used to describe the Sun's gravitational field and advances of planetary perihelia in order to obtain upper bounds on the allowed $\mathrm{f}(\mathrm{T})$ corrections. In [11], the perihelion precession of planetary orbits is estimated for different gravity theories in stringinspired models. Moreover, a way to obtain information about higher dimensions from observations by studying a brane based spherically symmetric solution is considered for the classic tests of General Relativity in [12]. The analytical computation of the Mercury perihelion precession in the frame of relativistic gravitational law and comparison with general relativity is presented in [13]. The direct computation of the perihelion shift meets a certain difficulties due to the fact that the geodesics equations in RG are nonlinear, and therefore cannot be solved exactly in general case. For instance, the geodesic equations resulting from the Schwarzschild gravitational metric element are solved exactly by the Weierstra $\beta$ - Jacobi modular form [14]. Mostly, the perihelion precession of planetary orbits based on Einstein's equations had been calculated in different approximations for a general spherically symmetric line element. Note, however, that even after the approximate solution for the orbit is found, the problem of calculating the perihelion shift has still to be solved. Generally, such a calculation is performed from the assumption of its smallness or the smallness of the orbital eccentricity. However, in work [15] it has been shown that the calculations of such a kind can be performed without an assumption of existence of a small physical parameter, with any desired accuracy by means of the so-called homotopy perturbation method (HPM).

The idea of HPM which is a semi-analytical method was proposed for solving differential and integral equations [16], [17]. Later, the method is applied to solve the non-linear and non-homogeneous partial differential equations. This method has a significant advantage providing an analytical approximate solution to a wide range of nonlinear problems of the fundamental and applied sciences.It is interesting that this method sometimes allows to find even an exact solution with the help of a few iteration. This method and a wide spectrum of its application have been extensively developed for several years by numerous authors (see [18] and references therein).

Recently there were studies in which this method was used for analytical calculations in the field of cosmology and astrophysics (see, e.g. [19] - [22]). Our aim is to give one more application of the method to the problem of planetary motion in the spherically symmetric gravitational field in General Relativity. By applying this method, we obtain a simple formula for the angle of precession avoiding any restrictions on the smallness of physical parameters.

As an example, we consider the standard Schwarzschild and Reissner-Nordström metrics in order to find the perihelion precession angle via HPM formula. Finally, we obtain the perihelion shift in the gravitational field of a black hole surrounded by quintessence (Kiselev black hole) [23]. The main aim from this paper is to propose HPM to find the approximate values of precession for planetary motion in GR. Thus, we demonstrate that this method can proved the effectiveness in the calculation of some essential parameters in astrophysical problems. 


\section{Preliminaries}

In this section, we give some remarks on the calculation of precession angle from the approximate solutions for the orbits, and the main ideas of HPM which are required for solving our problem.

\subsection{Calculation of the precession angle from approxi- mate solution for the orbits}

The calculation of the precession angle would be a simple problem if one knew the exact solution of the equations of motion. However, except in rare cases, the orbital motion can be described only in a certain approximation, for example, in the following form

$$
\mathrm{u}(\phi)=\frac{\mathrm{M}}{\mathrm{L}^{2}}(1+\mathrm{e} \cos \phi)+\frac{\mathrm{M}}{\mathrm{L}^{2}} \mathrm{U}(\phi),
$$

Where the function $\mathrm{u}=1 / \mathrm{r}, \mathrm{M}$ is the mass of the central star, $\mathrm{L}$ is the conserved angular momentum of the planet, e is the orbital eccentricity, and $\mathrm{U}(\phi)$ is a differentiable function of angle $\phi$ and the orbital parameters, which gives a correction compared to the Keplerian orbit determined by the first term of equation (1). Finding the correction $\mathrm{U}(\phi)$ has been widely discussed (see, e.g., [2]). We only note that one of the authors of the present paper (V.K.S.) have shown that the correction function $\mathrm{U}(\phi)$ can be alternatively obtained by a simple calculation with the help of HPM [20] or the He's variational iteration method [24].

In any case, after finding the corrections, the problem of calculation of the perihelion displacement angle from (1) could be solved The simplest way to find the angle from this equation follows from several assumptions concerned to the smallness of orbital parameters, such as e , M/L and so on [2]. It allows to represent equation (1) in an approximate form as follows

$$
\mathrm{u}(\phi) \approx \frac{\mathrm{M}}{\mathrm{L}^{2}}[1+\mathrm{e} \cos (\phi-\varepsilon \phi)] .
$$

For the perihelion of orbit, it satisfies $\cos (\phi-\varepsilon \phi)=1$, and hence $\phi=2 \pi+2 \pi \varepsilon$. Therefore, the precession angle of perihelion is $\alpha=2 \pi \varepsilon$.

A more precise formula for the shift angle can be obtained from the maximum condition $\mathrm{u}^{\prime}(\phi)=0$ in the perihelion. Applying this condition to equation (1), one can get the following equation

$$
\sin \phi-\mathrm{e}^{-1} \mathrm{U}^{\prime}(\phi)=0
$$

To be solved subject to the unperturbed solution $\phi=2 \pi$. This equation could be approximately solved using the assumption that the precession angle $\alpha$ is much smaller compared to $2 \pi$, i.e. using the approximate equalities $\sin (2 \pi+\alpha) \approx \alpha, \cos (2 \pi+\alpha) \approx 1$, and the similar ones.

Nevertheless, when the need in greater accuracy arises, one has to take into account not only linear terms but also the higher degrees of $\alpha$ in expansion of $\mathrm{U}^{\prime}(2 \pi+\alpha)$ in the power series. This approach leads to the difficult problem of solving the algebraic equation with respect to $\alpha$. As a result, one has to simplify this calculation once again according to the relevant physical parameters of the problem. This excludes a large value of displacement, which can be caused by the real physical sources of gravity. In this paper. we continue to use HPM to the astrophysical problems, demonstrating how one can find the precession angle from equation (3) without any small physical parameters.

However, we would like to note that one of the simplest methods for finding successively better approximations to the roots of equation (3), known as Newton's method, could be also applied in this case. If we denote the 1.h.s of equation (3) as $\mathrm{f}(\phi)=\sin \phi-\mathrm{e}^{-1} \mathrm{U}^{\prime}(\phi)$, the Newton's iteration for the equation $\mathrm{f}(\phi)=0$ can be represented as follows $\phi_{\mathrm{n}+1}=\phi_{\mathrm{n}}-\frac{\mathrm{f}\left(\phi_{\mathrm{n}}\right)}{\mathrm{f}^{\prime}\left(\phi_{\mathrm{n}}\right)}$. Taking $\phi_{\mathrm{n}}=2 \pi$, we can obtain the following first-order approximation for the precession angle $\alpha=\phi_{n+1}-2 \pi$ :

$\alpha_{N}=\frac{e^{-1} U^{\prime}(2 \pi)}{1-e^{-1} U^{\prime \prime}(2 \pi)}$

\subsection{Briefly on HPM}

Let us recall the basic ideas of HPM [16] for solving nonlinear equations. Here, we would like to consider the functional equation:

$\mathrm{A}(\phi)=\mathrm{g}(\mathrm{r}), \mathrm{r} \in \Omega$,

With the boundary conditions $\mathrm{B}(\phi, \partial \phi / \partial \mathrm{n})=0 ; \mathrm{r} \in \Gamma$, where $\mathrm{A}$ is a general operator, B is a boundary operator, $\mathrm{g}(\mathrm{r})$ is a known analytic function, $\Gamma$ is the boundary of the domain $\Omega$. As always, we suppose that operator A can be divided into two parts: $\mathrm{R}$ and $\mathrm{N}$. Therefore, equation (5) takes the following form:

$\mathrm{R}(\phi)+\mathrm{N}(\phi)=\mathrm{g}(\mathrm{r})$

Following the homotopy technique, we have to construct a homotopy $\phi(r, p): \Omega \times[0,1] \rightarrow \Re$, say[18],

$\mathrm{H}(\phi, \mathrm{p})=(1-\mathrm{p})\left[\mathrm{R}(\phi)-\mathrm{R}\left(\phi_{0}\right)\right]+\mathrm{p}[\mathrm{A}(\phi)-\mathrm{g}(\phi)]=0$,

Where $\mathrm{r} \in \Omega, \mathrm{p} \in[0,1]$ is an imbedding parameter, and $\phi_{0}$ is an initial approximation of (5). Hence, one can see that

$\mathrm{H}(\phi, 0)=\mathrm{R}(\phi)-\mathrm{R}\left(\phi_{0}\right)=0$,

$\mathrm{H}(\phi, 1)=\mathrm{A}(\phi)-\mathrm{g}(\mathrm{r})=0$,

And changing $p$ from 0 to 1 is the same as changing $H(\phi, p)$ from $\mathrm{R}(\phi)-\mathrm{R}\left(\phi_{0}\right)$ to $\mathrm{A}(\phi)-\mathrm{g}(\mathrm{r})$, which are called homotopic. In topology, this is called deformation. Due to the fact that $0 \leq p \leq 1$ can be considered as a small parameter, by applying the perturbation procedure, one can assume that the solution of (7) can be expressed as a series in $p$, as follows: $\phi=\phi_{0}+\mathrm{p} \phi_{1}+\mathrm{p}^{2} \phi_{2}+\mathrm{p}^{3} \phi_{3}+\ldots$. When we put $\mathrm{p} \rightarrow 1$, then equation (7) corresponds to (6), and the approximate solution for (6) becomes as follows

$$
\phi=\lim _{\mathrm{p} \rightarrow 1} \phi=\phi_{0}+\phi_{1}+\phi_{2}+\phi_{3}+\ldots . .
$$

This series is convergent for the most cases. However, the convergent rate depends upon the nonlinear operator $\mathrm{A}(\phi)$. Sometimes, even the first approximation is sufficient to obtain the exact solution [16]. For the details about the convergence of HPM, we refer the reader to Refs. [25] and [26].

As it is emphasized in, the second derivative of $\mathrm{N}(\phi)$ with respect to $\phi$ must be small, because the parameter $\mathrm{p}$ may be relatively large, i.e. $\mathrm{p} \rightarrow 1$, and the norm of $\mathrm{R}^{-1} \partial \mathrm{N} / \partial \phi$ must be smaller than one, in order that the series converges.

\section{Calculation of precession angle by HPM}

Since for finding the precession angle it is necessary to solve nonlinear equation $u^{\prime}(\phi)=0$ in the form (3), one can apply HPM for 
this purpose. Note that the correct approximate solution represented in the form (3) has to satisfy $\mathrm{U}^{\prime}(0)=0$. Thus, let us consider the following homotopy for equation (3)

$$
\sin \phi-p \mathrm{e}^{-1} \mathrm{U}^{\prime}(\phi)=0,
$$

Where $\mathrm{p} \in[0,1]$ is the imbedding parameter. According to HPM, we assume that the solution for (10) can be represented by a series in $\mathrm{p}$, that is

$$
\phi=\phi_{0}+\mathrm{p} \phi_{1}+\mathrm{p}^{2} \phi_{2}+\mathrm{p}^{3} \phi_{3}+\ldots
$$

As $\mathrm{p} \rightarrow 1$, equation (10) tends to (3), and (11) becomes the approximate solution of (3), that is $\phi=\lim _{\mathrm{p} \rightarrow 1} \phi=\phi_{0}+\phi_{1}+\phi_{2}+\phi_{3}+\ldots$.

Next, we have to express both terms in equation (10) in the form of Taylor series in $\mathrm{p}$ as

$$
\begin{aligned}
& \sin \phi=\sin \phi_{0}+\mathrm{p} \phi_{1} \cos \phi_{0}+\mathrm{p}^{2}\left(\phi_{2} \cos \phi_{0}-\frac{1}{2} \phi_{1}^{2} \sin \phi_{0}\right) \\
& +\mathrm{p}^{3}\left(\phi_{3} \cos \phi_{0}-\phi_{1} \phi_{2} \sin \phi_{0}-\frac{1}{6} \phi_{1}^{3} \cos \phi_{0}\right)+\ldots
\end{aligned}
$$

And

$$
\mathrm{U}^{\prime}(\phi)=\mathrm{U}^{\prime}\left(\phi_{0}\right)+\phi_{1} \mathrm{U}^{\prime \prime}\left(\phi_{0}\right)+\mathrm{p}^{2}\left[\phi_{2} \mathrm{U}^{\prime \prime}\left(\phi_{0}\right)+\frac{1}{2} \phi_{1}^{2} \mathrm{U}^{\prime \prime \prime}\left(\phi_{0}\right)\right]+\ldots
$$

Substituting (12) and (33) into equation (10), we get the following set of simple algebraic equations

$$
\begin{aligned}
& \mathrm{p}^{0}: \quad \sin \phi_{0}=0, \\
& \mathrm{p}^{1}: \quad \phi_{1} \cos \phi_{0}-\mathrm{e}^{-1} \mathrm{U}^{\prime}\left(\phi_{0}\right)=0, \\
& \mathrm{p}^{2}: \quad \phi_{2} \cos \phi_{0}-\frac{1}{2} \phi_{1}^{2} \sin \phi_{0}-\mathrm{e}^{-1} \phi_{1} \mathrm{U}^{\prime \prime}\left(\phi_{0}\right)=0, \\
& \mathrm{p}^{3}: \quad \phi_{3} \cos \phi_{0}-\phi_{1} \phi_{2} \sin \phi_{0}-\frac{1}{6} \phi_{1}^{3} \cos \phi_{0} \\
& -\mathrm{e}^{-1}\left[\phi_{2} \mathrm{U}^{\prime \prime}\left(\phi_{0}\right)+\frac{1}{2} \phi_{1}^{2} \mathrm{U}^{\prime \prime}\left(\phi_{0}\right)\right]=0,
\end{aligned}
$$

Since we consider the Keplerian orbit as the path of unperturbed motion, we have to take $\phi_{0}=2 \pi$ for the solution of equation (14). Therefore, the precession angle is given by $\alpha_{\mathrm{HPM}}=\phi_{1}+\phi_{2}+\phi_{3}+\ldots$ as it follows from (9) at $\mathrm{p} \rightarrow 1$. Solving equations (15)-(17), one can obtain

$$
\begin{aligned}
& \alpha_{H P M} \approx \mathrm{e}^{-1} \mathrm{U}^{\prime}(2 \pi)\left(1+\mathrm{e}^{-1} \mathrm{U}^{\prime \prime}(2 \pi)\right. \\
& \left.+\mathrm{e}^{-2}\left[\mathrm{U}^{\prime \prime 2}(2 \pi)+\frac{\mathrm{U}^{\prime}(2 \pi) \mathrm{U}^{\prime \prime \prime}(2 \pi)}{2}+\frac{\mathrm{U}^{\prime 2}(2 \pi)}{6}\right]\right) .
\end{aligned}
$$

The obvious advantage of this formula is the absence of any requirement for smallness of the angle. At the same time, the number of terms taken into account when calculating $\alpha$ by this equation determines only the accuracy with which we find out this angle. If necessary, the subsequent terms in the approximate formula (18) can be easily obtained by the corresponding extension of series in equations (12) and (13). Note that the final accuracy with which the the angle $\alpha$ can be find out from equation (18) is determined in a great degree by the accuracy of the approximate equation (1)

One can easily see that equation (4) gives the same value of angle as it represented by equation (18) in the first-order approximation only if $\left|\mathrm{e}^{-1} \mathrm{U}^{\prime \prime}(2 \pi)\right|\langle\langle 1 \quad$, that is when $\left(1-\mathrm{e}^{-1} \mathrm{U}^{\prime \prime}(2 \pi)\right)^{-1} \approx 1+\mathrm{e}^{-1} \mathrm{U}^{\prime \prime}(2 \pi)$. Noteworthy that in the derivation of equation (24), the restriction of such a kind is not used.

\section{Some examples of the precession angle}

Taking the order of accuracy represented by equation (18), let us calculate the magnitudes of the precession angles in some relevant cases of a spherically symmetric spacetime. Since the planetary orbits in GR are usually treated as the time-like geodesic in the curved spacetime [1], [2], we should consider the approximate solutions (3) for the geodesic equation in the spherically symmetric spacetime,

$\frac{d^{2} x^{\mu}}{d \tau^{2}}+\Gamma_{v \sigma}^{\mu} \frac{d x^{\nu}}{d \tau} \frac{d x^{\sigma}}{d \tau}=0$

In the spherical coordinates $x^{\mu}=(t, r, \theta, \phi)$. The general stationary spherically symmetric spacetime line element is represented by

$\mathrm{ds}^{2}=-\mathrm{f}(\mathrm{r}) \mathrm{dt} \mathrm{t}^{2}+\frac{\mathrm{dr}^{2}}{\mathrm{~h}(\mathrm{r})}+\mathrm{r}^{2}\left(\mathrm{~d} \theta^{2}+\sin ^{2} \theta \mathrm{d} \phi^{2}\right)$

It is well known that the perihelion precession is usually related to the geodesics orbits, i.e. $r(\phi)$, and the coordinate $u \equiv 1 / r$ is more convenient than $r$ to derive the perihelion precession. The main equation of such a motion could be simply obtained from equation (19) as follows [2]

$$
\frac{d^{2} u}{d \phi^{2}}=\frac{E^{2}}{2 L^{2}} \frac{d}{d u}\left[\frac{h(u)}{f(u)}\right]-h(u) u-\frac{1}{2}\left(\frac{1}{L^{2}}+u^{2}\right) \frac{d h(u)}{d u},
$$

Where $E=f(r)(d t / d \tau)$ is the total energy of the planet, and $\mathrm{L}=\mathrm{r}^{2}(\mathrm{~d} \phi / \mathrm{d} \tau)$ is its angular momentum per unit mass.

Below, we apply HPM formula (18) for the solutions of this equation in the form (3) obtained earlier by several authors.

\subsection{Schwarzschild metric}

In the simplest case of the Schwarzschild metric describing the gravitational field of an uncharged non-rotating star, we have $f(r)=h(r)=1-2 M / r$ in equation (19), where $M$ is the mass of star. Therefore, one can find that the geodesics equation (20) in Schwarzschild spacetime reduces to the following equation

$\frac{\mathrm{d}^{2} \mathrm{u}}{\mathrm{d} \phi^{2}}+\mathrm{u}=\frac{\mathrm{M}}{\mathrm{L}^{2}}+3 \mathrm{Mu}^{2}$

The approximate solution $\mathrm{u}(\phi)$ of this equation can be represented (see, e.g.,[20, 24]) by

$$
\begin{aligned}
& \mathrm{u}(\phi)=\frac{\mathrm{M}}{\mathrm{L}^{2}}(1+\mathrm{e} \cos \phi)+\frac{\mathrm{M}^{3}}{\mathrm{~L}^{4}}\left[3+2 \mathrm{e}^{2}+3 \mathrm{e} \phi \sin \phi\right. \\
& \left.-\mathrm{e}^{2} \cos ^{2} \phi-\left(3+\mathrm{e}^{2}\right) \cos \phi\right] .
\end{aligned}
$$

Comparing equations (1) and (21), we have in this case that

$$
\mathrm{U}(\phi)=\frac{\mathrm{M}^{2}}{\mathrm{~L}^{2}}\left[3+2 \mathrm{e}^{2}+3 \mathrm{e} \phi \sin \phi-\mathrm{e}^{2} \cos ^{2} \phi-\left(3+\mathrm{e}^{2}\right) \cos \phi\right],
$$


And, hence,

$$
\begin{aligned}
& \mathrm{U}^{\prime}(2 \pi)=-\mathrm{U}^{\prime \prime \prime}(2 \pi)=6 \pi \mathrm{e} \frac{\mathrm{M}^{2}}{\mathrm{~L}^{2}}, \\
& \mathrm{U}^{\prime \prime}(2 \pi)=3(1+\mathrm{e})^{2} \frac{\mathrm{M}^{2}}{\mathrm{~L}^{2}} .
\end{aligned}
$$

Using these expressions in (18), we obtain the following angle of precession in HPM approximation

$$
\alpha_{H P M}=\frac{6 \pi \mathrm{M}^{2}}{\mathrm{~L}^{2}}\left[1+\frac{3(1+\mathrm{e})^{2}}{\mathrm{e}} \frac{\mathrm{M}^{2}}{\mathrm{~L}^{2}}+\left(\frac{9(1+\mathrm{e})^{4}}{\mathrm{e}^{2}}-12 \pi^{2}\right) \frac{\mathrm{M}^{4}}{\mathrm{~L}^{4}}\right] .
$$

At the same time, the precession angle (4) obtained via the Newton's iteration method follows from (23) as

$$
\alpha_{N}=6 \pi \frac{M^{2}}{L^{2}}\left(1-\frac{3(1+e)^{2}}{e} \frac{M^{2}}{L^{2}}\right)^{-1}
$$

This equation yields the same value of angle as it represented by equation (24) in the first-order approximation when $\frac{3(1+e)^{2}}{\mathrm{e}} \frac{\mathrm{M}^{2}}{\mathrm{~L}^{2}}\langle\langle 1$ Fig. 1 shows the dependence of the precession angle on the parameter $\mathrm{M} / \mathrm{L}$ for the Einstein's formula $6 \pi \mathrm{M}^{2} / \mathrm{L}^{2}$ and for the approximate values of shift according to equations (24) and (25). As can be seen, formula (25) provides a significant divergence for the large values of parameter, while formula (24) is free from such a divergence.

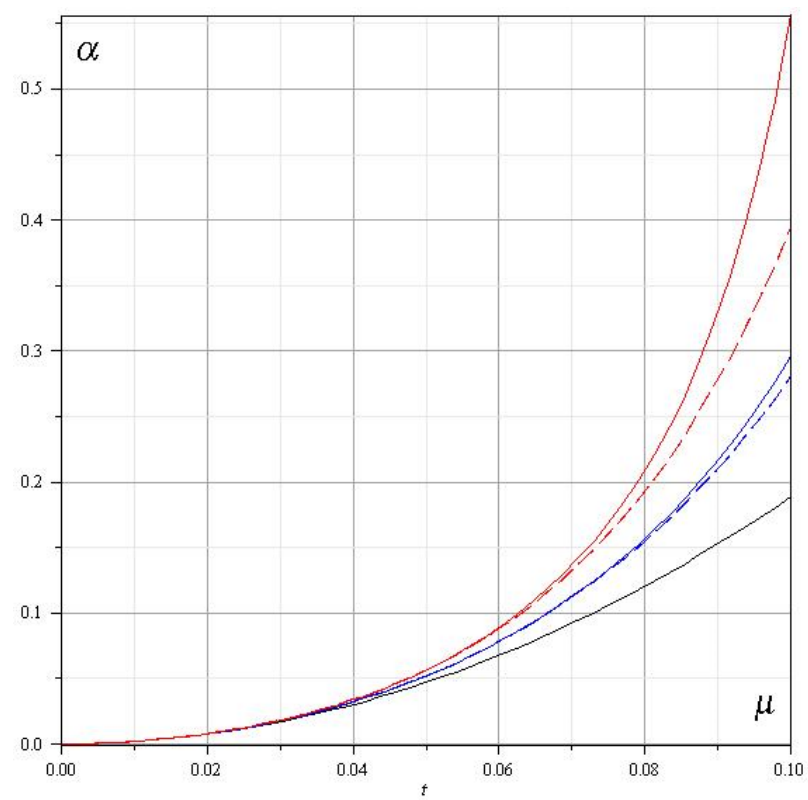

Fig. 1: Shows the Einstein's Precession Angle $\alpha_{\mathrm{E}}=6 \pi \mathrm{M}^{2} / \mathrm{L}^{2}$ (Black Line), and the Angle $\alpha_{\mathrm{HPM}}$ in (24) (Blue Lines) and $\alpha_{\mathrm{N}}$ In (25) (Red Lines) Versus the Parameter $\mu=\mathrm{M} / \mathrm{L}$. Here the Eccentricity Is Equal To $\mathrm{e}=0.1$ (Solid Lines) or e $=0.05$ (Dashed Lines).

\subsection{Reissner-nordström spacetime}

In the case of the Reissner-Nordstrц $m$ spacetime of a charged star, we have [1]

$$
f(u)=h(u)=1-2 M u+Q^{2} u^{2},
$$

Where $\mathrm{Q}$ is the charge of star. According to (26), the geodesic equation (20) is the following one

$\frac{\mathrm{d}^{2} \mathrm{u}}{\mathrm{d} \phi^{2}}+\left(1+\frac{\mathrm{Q}^{2}}{\mathrm{~L}^{2}}\right) \mathrm{u}=\frac{\mathrm{M}}{\mathrm{L}^{2}}+3 \mathrm{u}^{2} \mathrm{M}-2 \mathrm{Q}^{2} \mathrm{u}^{3}$

The simplest approximate solution of equation (27) can be given by (see, e.g., [24])

$$
\begin{aligned}
& \mathrm{u}(\phi)=\frac{\mathrm{M}}{\mathrm{L}^{2}}(1+\mathrm{e} \cos \phi)+\frac{\mathrm{M}^{3}}{\mathrm{~L}^{4}}\left[3+2 \mathrm{e}^{2}-2\left(1+2 \mathrm{e}^{2}\right) \frac{\mathrm{Q}^{2}}{\mathrm{~L}^{2}}\right. \\
& -\frac{\mathrm{Q}^{2}}{\mathrm{M}^{2}}+\left(1-\frac{\mathrm{Q}^{2}}{\mathrm{~L}^{2}}+\frac{\mathrm{Q}^{2}}{6 \mathrm{M}^{2}}-\mathrm{e}^{2} \frac{\mathrm{Q}^{2}}{4 \mathrm{~L}^{2}}\right) 3 \mathrm{e} \phi \sin \phi \\
& -\left(3+\mathrm{e}^{2}+\left(\mathrm{e}^{3}-8 \mathrm{e}^{2}-8\right) \frac{\mathrm{Q}^{2}}{4 \mathrm{~L}^{2}}-\frac{\mathrm{Q}^{2}}{\mathrm{M}^{2}}\right) \cos \phi \\
& \left.-\left(1-2 \frac{\mathrm{Q}^{2}}{\mathrm{~L}^{2}}\right) \mathrm{e}^{2} \cos ^{2} \phi+\frac{\mathrm{Q}^{2}}{4 \mathrm{~L}^{2}} \mathrm{e}^{3} \cos ^{3} \phi\right] .
\end{aligned}
$$

Comparing this solution with (1) in this case, one can get all terms in equation (18), for example,

$$
\mathrm{U}^{\prime}(2 \pi)=6 \pi \mathrm{e} \frac{\mathrm{M}^{2}}{\mathrm{~L}^{2}}+\pi \mathrm{e} \frac{\mathrm{Q}^{2}}{\mathrm{~L}^{2}}-3 \pi \mathrm{e}\left(4+\mathrm{e}^{2}\right) \frac{\mathrm{M}^{2} \mathrm{Q}^{2}}{2 \mathrm{~L}^{4}} .
$$

With the help of (18), one can easily obtain the value of shift angle in the minimum degree of approximation as

$$
\alpha=6 \pi \frac{M^{2}}{L^{2}}+\pi \frac{Q^{2}}{L^{2}}-3 \pi\left(4+e^{2}\right) \frac{M^{2} Q^{2}}{2 L^{4}} .
$$

We do not provide here the full calculation according to the HPM formula (18) only due to its cumbersome nature. Despite that we have deliberately taken the minimum degree of approximation in this calculation, all subsequent approximations can be readily obtained.

\subsection{Precession angle in the gravitational field of Kiselev black hole}

The spherically symmetric solutions describing a black hole surrounded by dark energy in the form of a quintessential scalar field with equation of state in the form $\mathrm{p}=\mathrm{w}_{\mathrm{q}} \rho$, with the quintessential parameter $-1<\mathrm{w}_{\mathrm{q}}<-1 / 3$, has been found by Kiselev [23]. The geometry of the static spherically symmetric black hole surrounded by a quintessence (or Kiselev spacetime) is given by equation (1) with [23]

$$
f(u)=h(u)=1-2 M u-\sigma u^{3 w_{q}+1},
$$

Where $M$ is the mass of the black hole and $\sigma$ is the quintessence parameter related to the energy density as follows

$\rho_{\mathrm{q}}=-3 \sigma \mathrm{w}_{\mathrm{q}} \mathrm{u}^{3\left(1+\mathrm{w}_{\mathrm{q}}\right)} / 2$

Substituting (31) into equation (20), one can obtain the following geodesic equation in the gravitational field of Kiselev black hole 


$$
\begin{aligned}
& \frac{d^{2} u}{d \phi^{2}}+u=\frac{M}{L^{2}}+3 u^{2} M \\
& +\frac{\sigma\left(3 w_{q}+1\right)}{2 L^{2}} u^{3 w_{q}}+\frac{3 \sigma\left(w_{q}+1\right)}{2} u^{3 w_{q}+2} .
\end{aligned}
$$

The approximate solution for equation (32) in the case $\mathrm{w}_{\mathrm{q}}=-1 / 3$ is obtained in [24] in the form (1) with

$$
\begin{aligned}
& \mathrm{U}(\phi)=\frac{\mathrm{M}^{2}}{\mathrm{~L}^{2}}\left[3+2 \mathrm{e}^{2}+\sigma \frac{\mathrm{L}^{2}}{\mathrm{M}^{2}}-\mathrm{e}^{2} \cos ^{2} \phi\right. \\
& \left.+3 \mathrm{e}\left(1+\frac{\sigma}{6} \frac{\mathrm{L}^{2}}{\mathrm{M}^{2}}\right) \phi \sin \phi-\left(3+\mathrm{e}^{2}+\sigma \frac{\mathrm{L}^{2}}{\mathrm{M}^{2}}\right) \cos \phi\right]
\end{aligned}
$$

And, hence,

$$
\begin{aligned}
& \mathrm{U}^{\prime}(2 \pi)=-\mathrm{U}^{\prime \prime \prime}(2 \pi)=6 \pi \mathrm{e} \frac{\mathrm{M}^{2}}{\mathrm{~L}^{2}}+\pi \mathrm{e} \sigma, \\
& \mathrm{U}^{\prime \prime}(2 \pi)=3(1+\mathrm{e})^{2} \frac{\mathrm{M}^{2}}{\mathrm{~L}^{2}}+(1+\mathrm{e}) \sigma .
\end{aligned}
$$

Using these expressions in (18), we obtain the following angle of precession in HPM approximation

$$
\begin{aligned}
& \alpha_{H P M}=\left(6 \pi \frac{\mathrm{M}^{2}}{\mathrm{~L}^{2}}+\pi \sigma\right)\left[1+\frac{(1+\mathrm{e})}{\mathrm{e}} \sigma+\frac{3(1+\mathrm{e})^{2}}{\mathrm{e}} \frac{\mathrm{M}^{2}}{\mathrm{~L}^{2}}\right. \\
& +\left(\frac{3(1+\mathrm{e})^{2}}{\mathrm{e}^{2}}-\pi^{2}\right) \frac{\sigma^{2}}{3}+2\left(\frac{3(1+\mathrm{e})^{3}}{\mathrm{e}^{2}}-2 \pi^{2}\right) \frac{\sigma \mathrm{M}^{2}}{\mathrm{~L}^{2}} \\
& \left.+\left(\frac{9(1+\mathrm{e})^{4}}{\mathrm{e}^{2}}-12 \pi^{2}\right) \frac{\mathrm{M}^{4}}{\mathrm{~L}^{4}}\right],
\end{aligned}
$$

Which coincides with (24) at $\sigma=0$. At the same time, the approximate magnitude of the precession angle followed from equations (4) and (34) is given by

$\alpha_{N}=\left(6 \pi \frac{\mathrm{M}^{2}}{\mathrm{~L}^{2}}+\pi \sigma\right)\left[1-\frac{(1+\mathrm{e})}{\mathrm{e}} \sigma-\frac{3(1+\mathrm{e})^{2}}{\mathrm{e}} \frac{\mathrm{M}^{2}}{\mathrm{~L}^{2}}\right]^{-1}$.

Provided that $\sigma<\left\langle 1\right.$ and $\left(\mathrm{M}^{2} / \mathrm{L}^{2}\right)\langle<1$, both equations (34) and (36) are substantially simplified up to

$$
\alpha \approx 6 \pi \frac{\mathrm{M}^{2}}{\mathrm{~L}^{2}}+\pi \sigma .
$$

For the specific value of eccentricity $\mathrm{e}=0.12$, the precession angle in different approximations given by equations (35) - (37) as a function of $\mu=\mathrm{M} / \mathrm{L}$ and $\sigma$ is presented in Fig. 2 .

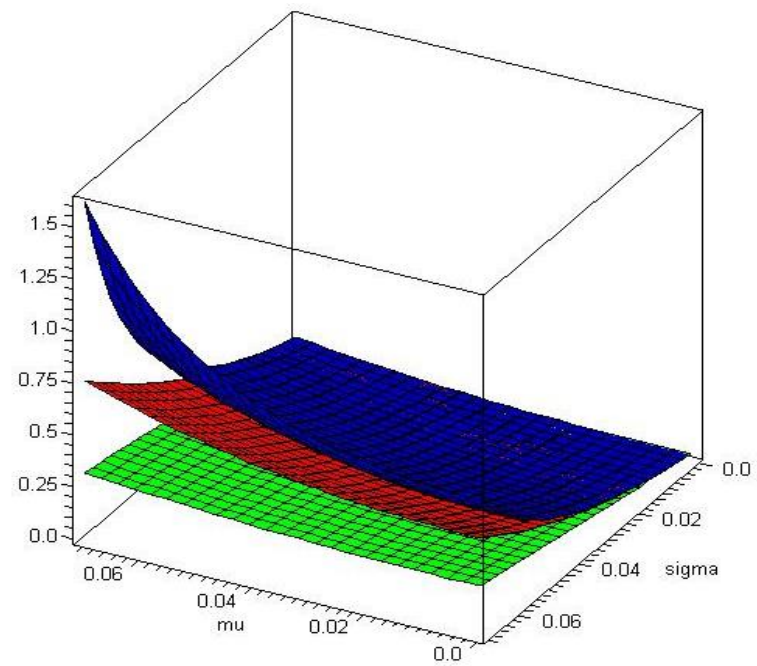

Fig. 2: Shows the Precession Angle in the Gravitational Field of Kiselev Black Hole According to Formulae (35) (In Red), (36) (in Blue) and (37) (In Green) Versus the Parameters $\mu=\mathrm{M} / \mathrm{L}$ and $\sigma$. Here the Eccentricity Is Taken to $\mathrm{Be} \mathrm{e}=0.12$.

\section{Conclusion}

In this paper, we have shown that HPM can be employed to obtain the approximate value of the precession angle of the planetary orbit in the framework of GR mechanics. Using the main idea of HPM, we have constructed the appropriate homotopy that leads to the problem of solving the set of linear algebraic equations. As a result, we have obtained a simple formula for the angle of precession avoiding any restrictions on the smallness of the physical parameters. For the illustrative purpose, we have considered the examples of analytic calculation of the precession angle in the Schwarzschild metric and the Reissner-Nordström spacetime of a charged star using the approximate geodesics solutions obtained earlier. Moreover, we have applied HPM to obtain the perihelion shift due to the gravity of Kiselev black hole subject to the equation of state $\mathrm{w}_{\mathrm{q}}=-1 / 3$. It is worthy to note, that all these results could be obtained by a minimum size of computations.

According to our results, it can be concluded that HPM is working well for the approximate solutions for the perihelion shift of planetary motion around the central mass. Foremost, the undoubted advantage of this method consists of that there is no need to establish a small parameter for solving a problem in some approximation, because such a small parameter sometimes could destroy the main feature of the exact solution. On the other hand, the approximate solution and the rate of its convergence in this method greatly depend on the accuracy of the approximate solution for the geodesics motion. Moreover, it must be acknowledged that the formula (18) works only within the framework of the values of physical parameters for which the approximation (3) is valid.

\section{References}

[1] S. Weinberg. Gravitation and Cosmology: Principles and Applications of the General Theory of Relativity, John Wiley. Press, New York, 1972

[2] Ya-Peng Hu, Hongsheng Zhang, Jun-Peng Hou, and Liang-Zun Tang, "Perihelion Precession and Deflection of Light in the General Spherically Symmetric Spacetime", Advances in High Energy Physics, Volume 2014, Article ID 604321, 7 pages. https://doi.org/10.1155/2014/604321.

[3] Christian Magnan, "Complete calculations of the perihelion precession of Mercury and the deflection of light by the Sun in General Relativity", http://arxiv.org/abs/0712.3709

[4] Hideyoshi Arakida, "Note on the Perihelion Periastron Advance Due to Cosmological Constant", International Journal of Theoretical Physics, 52 (2013), 1408. https://doi.org/10.1007/s10773-0121458-2. 
[5] A. A. Vankov, "General Relativity Problem of Mercury's Perihelion Advance Revisite", http://arxiv.org/abs/1008.1811

[6] G. V. Kraniotis, S. B. Whitehouse, "Compact calculation of the Perihelion Precession of Mercury in General Relativity, the Cosmological Constant and Jacobi's Inversion problem", Classical and Quantum Gravity, $20 \quad$ (2003), 4817-4835. https://doi.org/10.1088/0264-9381/20/22/007.

[7] A.S. Fokas, C.G. Vayenas, D. Grigoriou, "Analytical computation of the Mercury perihelion precession via the relativistic gravitational law and comparison with general relativity", http://arxiv.org/abs/1509.03326

[8] M. Pejic, "Calculating perihelion precession using the multiple scales method", http://math.berkeley.edu/ mpejic/pdfdocuments/PerihelionPrecession.pdf

[9] M. L. Ruggiero, "Perturbations of Keplerian orbits in stationary spherically symmetric spacetimes", International Journal of Modern Physsics D, 23(5) (2014), 1450049. https://doi.org/10.1142/S0218271814500497.

[10] L. Iorio, E. N. Saridakis, "Solar system constraints on $\mathrm{f}(\mathrm{T})$ gravity", Monthly Notices of the Royal Astronomical Society, 427 (2012), 1555. https://doi.org/10.1111/j.1365-2966.2012.21995.x.

[11] Sumanta Chakraborty, Soumitra SenGupta, "Solar system constraints on alternative gravity theories", Physical Review D, 89 (2014) 026003. https://doi.org/10.1103/PhysRevD.89.026003

[12] R. R. Cuzinatto, P. J. Pompeia, M. de Montigny, F. C. Khanna, J. M. Hoff da Silva, "Classic tests of General Relativity described by brane-based spherically symmetric solutions", European Physical Journal C 74 (2014), 3017. https://doi.org/10.1140/epjc/s10052014-3017-x.

[13] A.S. Fokas, C.G. Vayenas, D. Grigoriou, "Analytical computation of the Mercury perihelion precession via the relativistic gravitational law and comparison with general relativity", http://arxiv.org/abs/1509.03326.

[14] G. V. Kraniotis, S. B. Whitehouse, "Compact calculation of the Perihelion Precession of Mercury in General Relativity, the Cosmological Constant and Jacobi's Inversion problem", Classical and Quantum Gravity, 20 (2003), 4817-4835. https://doi.org/10.1088/0264-9381/20/22/007.

[15] V. K. Shchigolev, D. N. Bezbatko, "Studying Gravitational Deflection of Light by Kiselev Black Hole via Homotopy Perturbation Method", http://arxiv.org/abs/1612.07279

[16] J.-H. He, "Homotopy perturbation technique", Computer Methods in Applied Mechanics and Engineering, 178 (1999), 257-262. https://doi.org/10.1016/S0045-7825(99)00018-3.

[17] J.-H. He, "A coupling method of homotopy technique and perturbation technique for nonlinear problems", International Journal of Non-Linear Mechanics, 35 (1) (2000), 37-43. https://doi.org/10.1016/S0020-7462(98)00085-7.

[18] J.-H. He, "Asymptotic Methods for Solitary Solutions and Compactions", Abstract and Applied Analysis, Volume 2012, Article ID 916793, 130 pages. https://doi.org/10.1155/2012/916793.

[19] V. Shchigolev, "Homotopy Perturbation Method for Solving a Spatially Flat FRW Cosmological Model", Universal Journal of Applied Mathematics, 2(2) (2014), 99-103.

[20] V. K. Shchigolev, "Analytical Computation of the Perihelion Precession in General Relativity via the Homotopy Perturbation Method", Universal Journal of Computational Mathematics, 3(4) (2015), 45-49.

[21] F. Rahaman, S. Ray, A. Aziz, S. R. Chowdhury, D. Deb, "Exact Radiation Model For Perfect Fluid Under Maximum Entropy Principle", http://arxiv.org/abs/1504.05838

[22] Abdul Aziz, Saibal Ray, Farook Rahaman, "A generalized model for compact stars", European Physical Journal C, 76 (2016), 248. https://doi.org/10.1140/epjc/s10052-016-4090-0.

[23] V. V. Kiselev, "Quintessence and black holes", Classical and Quantum Gravity, 20 (2003), $1187-1198$. https://doi.org/10.1088/0264-9381/20/6/310.

[24] V. K. Shchigolev, "Variational iteration method for studying perihelion precession and deflection of light in General Relativity", In ternational Journal of Physical Research, 4 (2) (2016), 52-57. https://doi.org/10.14419/ijpr.v4i2.6530.

[25] J.-H. He, "A coupling method of homotopy technique and perturbation technique for nonlinear problems", International Journal of Non-Linear Mechanics, 35 (1) (2000), 37-43. https://doi.org/10.1016/S0020-7462(98)00085-7.

[26] L. Cveticanin, "Homotopy-perturbation method for pure nonlinear differential equation", Chaos, Solitons \& Fractals, 30(5) (2006) 1221 - 1230. https://doi.org/10.1016/j.chaos.2005.08.180. 